\title{
Analysis of Regulatory of Interrelated Activity of Hepatocyte and Hepatitis B Viruses
}

\author{
Mohiniso Baxromovna Hidirova ${ }^{*}$, Abrorjon Maxamatsoliyevich Turgunov \\ Scientific and Innovation Center of Information and Communication Technologies, Tashkent University of Information Technologies Named \\ After Muhammad Al-Khwarizmi, Tashkent, Uzbekistan
}

Email address:

mhidirova@yandex.ru (M. B. Hidirova), abrorjon-2017@mail.ru (A. M. Turgunov)

${ }^{*}$ Corresponding author

\section{To cite this article:}

Mohiniso Baxromovna Hidirova, Abrorjon Maxamatsoliyevich Turgunov. Analysis of Regulatory of Interrelated Activity of Hepatocyte and Hepatitis B Viruses. International Journal of Biomedical Materials Research. Vol. 6, No. 1, 2018, pp. 1-7. doi: 10.11648/j.ijbmr.20180601.11

Received: October 25, 2017; Accepted: November 16, 2017; Published: January 16, 2018

\begin{abstract}
In this article, we will present the results of the stability analysis of the equilibrium point of the mathematical model of the regulatory of the interrelated activity of the hepatocyte and hepatitis B viruses. The analysis of this model used the conditions of the Hayes criterion. In this study, the general condition of the Hayes criterion is obtained. If the general condition of the Hayes criterion is satisfied, then the equilibrium point is stable. If the general condition of the Hayes criterion is not fulfilled, then the equilibrium point is not stable, and hence thiscan describe modes "limit cycle", "chaos" and "black hole" mathematical models of the interrelated activity of the liver cell and hepatitis B viruses. The results of the computational experiment on the quantitative analysis of the regulatory of liver cell and HBV are presented.
\end{abstract}

Keywords: Regulatory, Mathematics Model, Equilibrium Points, Stability, Qualitative and Quantitative Analysis

\section{Introduction}

Hepatitis B is a viral infection that affects the liver. According to WHO estimates, 240 million people are chronically infected with the hepatitis B virus (HBV) [1]. Currently, this virus remains the third most common after diseases like cardiovascular system and oncological pathologies [2].

Approximately $19.4 \%$ of deaths in recent years have been caused by infections, and this is gradually increasing. The most deadly virus on the earth was various strains of the hepatitis virus, which kills more than 1.3 million people every year. The mortality from hepatitis increased by about $22 \%$ compared with HIV, tuberculosis and malaria. One of the reasons for this is that people do not even know that they have a virus. According to researchers, only $5 \%$ of developed and developing countries are aware of their diagnosis and treatment in medical institutions [3].

Hepatitis B virus (HBV) is one of the smallest enveloped DNA viruses that causes acute and chronic infections. HBV is able to evade the immune system of the host and persist lifelong within infected hepatocytes. During active replication, HBV produces enormous viral loads in the blood [4].

Therefore, it is necessary to study the functioning of hepatitis B in liver cell using methods of mathematical and computer modeling.

\section{Materials and Methods}

Mathematical modeling and model analysis of the dynamics of the hepatitis B virus are very important for the study of regulatory mechanisms and the dynamic behavior of the process of viral infection. Many scientific papers have proposed mathematical models describing the dynamics of viral hepatitis $\mathrm{B}$ in the liver cell. In these studies, mathematical modeling plays an important role in understanding and quantifying the biological mechanisms that control the dynamics of the hepatitis $B$ virus.

$\mathrm{Abu}$ O. and Onalo S. E. considered a mathematical model of the dynamics of transmission of the hepatitis B virus, which includes vaccination and treatment as control parameters. With the use of values of model parameters, the properties of the disease-free and the endemic equilibrium were numerically studied [5]. 
Moneim I. A. and Khalil H. A. studied the global behavior of HBV spread using the SEIR model with a constant vaccination rate. Infectivity during the incubation period is considered as the second mode of transmission [6].

These mathematical models describe the dynamics of the hepatitis B virus in the liver cell, at the cellular level. In accordance with the biological regularity of these processes, the development of an infectious disease occurs in the relationship between the genomes of the hepatitis B virus and liver cell. In the modeling of the regulatory of liver cell and hepatitis B virus, functional-differential equations were used. Equations are built on the basis of firmly established biological facts and regularities.

A mathematical model for the functioning of the regulatory of the hepatocyte (liver cell) and $\mathrm{HBV}$ at the molecular-genetic level has been developed by B. N. Hidirov $[7,8]$, and can be presented in the following form [9-12]:

$$
\begin{aligned}
& \varepsilon_{1} \frac{d X(t)}{d t}=\frac{a X^{2}(t-1)}{1+X^{2}(t-1)+c Y^{2}(t-1)}-X(t) ; \\
& \varepsilon_{2} \frac{d Y(t)}{d t}=\frac{b X(t-1) Y(t-1)}{1+d X^{2}(t-1)+Y^{2}(t-1)}-Y(t) ; \\
& X(t)=\varphi_{1}(t) ; Y(t)=\varphi_{2}(t) \text { at } t \in\left[t_{0} ; t_{0}+1\right] ;
\end{aligned}
$$

where $X(t), Y(t)$ - the values characterizing the activity of the molecular genetic systems of the liver cell and hepatitis B viruses, respectively; $\varepsilon_{1}, \varepsilon_{2}, a, b, c, d$ - parameters a regulatory of the model of the hepatocyte and $\mathrm{HBV} ; \varphi_{1}(t)$, $\varphi_{2}(t)-$ continuous functions on $\left[t_{0} ; t_{0}+1\right] ; t_{0}-$ the beginning of research; All parameters are positive.

This model describes the dynamics of the interrelated activity of liver cell and hepatitis B viruses at the moleculargenetic level. The system of functional-differential equations (1) is a nonlinear system and is a closed system. Therefore, we qualitatively investigate the equilibrium position of the (1).

To analyze the stability of the equilibrium position of (1), it is necessary to look at the equilibrium position of (1) around the equilibrium position [13]. By introducing small variable changes

$$
\begin{gathered}
X(t)=X_{0}+x(t) ; \\
Y(t)=Y_{0}+y(t) ; \\
X(t-1)=X_{0}+x(t-1) ; \\
Y(t-1)=Y_{0}+y(t-1),
\end{gathered}
$$

and we have the following

$$
\begin{aligned}
& \varepsilon_{1} \frac{d x(t)}{d t}=\frac{a\left(X_{0}+x(t-1)\right)^{2}}{1+\left(X_{0}+x(t-1)\right)^{2}+c\left(Y_{0}+y(t-1)\right)^{2}}-\left(X_{0}+x(t)\right) ; \\
& \varepsilon_{2} \frac{d y(t)}{d t}=\frac{b\left(X_{0}+x(t-1)\right)\left(Y_{0}+y(t-1)\right)}{1+d\left(X_{0}+x(t-1)\right)^{2}+\left(Y_{0}+y(t-1)\right)^{2}}-\left(Y_{0}+y(t)\right) .
\end{aligned}
$$

With the implementation of the simplification (2), we get the following equation:

$$
\begin{aligned}
& \varepsilon_{1} \frac{d x(t)}{d t}=\left(2-\frac{2}{a} X_{0}\right) x(t-1)-\frac{2 c}{a} Y_{0} y(t-1)-x(t) \\
& \varepsilon_{2} \frac{d y(t)}{d t}=\left(\frac{Y_{0}}{X_{0}}-\frac{2 d}{b} Y_{0}\right) x(t-1)+\left(1-\frac{2}{b} \frac{Y_{0}^{2}}{X_{0}}\right) y(t-1)-y(t) .
\end{aligned}
$$

This (3) is a linearized equation for small around equilibrium positions (1).

Let us find the characteristic equations for the linearized (3). To obtain the characteristic equations, we introduce the above expression on (4):

$$
\left|\begin{array}{cc}
\left.2-\frac{2}{a} X_{0}\right) e^{-\lambda}-1-\lambda \varepsilon_{1} & -\frac{2 c}{a} Y_{0} e^{-\lambda} \\
\left(\frac{Y_{0}}{X_{0}}-\frac{2 d}{b} Y_{0}\right) e^{-\lambda} & \left(1-\frac{2}{b} \frac{Y_{0}^{2}}{X_{0}}\right) e^{-\lambda}-1-\lambda \varepsilon_{2}
\end{array}\right|=0,
$$

and we have the characteristic equations (4). If $\frac{Y_{0}}{X_{0}}=\frac{2}{b c} Y_{0}$, then

$$
\begin{aligned}
& \left(2-\frac{2}{a} X_{0}\right) e^{-\lambda}-1-\lambda \varepsilon_{1}=0 ; \\
& \left(1-\frac{2}{b} \frac{Y_{0}^{2}}{X_{0}}\right) e^{-\lambda}-1-\lambda \varepsilon_{2}=0,
\end{aligned}
$$

we obtain (5) and (6).

First, for the analysis of (5), using the conditions of the Hayes criterion [14], it is necessary to arrive at the transcendental equation $(\lambda+a) e^{\lambda}+b=0$ :

$$
\left(\lambda+\frac{1}{\varepsilon_{1}}\right) e^{\lambda}+\left(\frac{2}{a} X_{0}-2\right) \frac{1}{\varepsilon_{1}}=0 .
$$

For the (7) we apply the condition of the Hayes criterion:

$$
\frac{1}{\varepsilon_{1}}>-1
$$

Given the positive value of the general parameters (1), then $\frac{1}{\varepsilon_{1}}>-1$ is discharged.

$$
\frac{1}{\varepsilon_{1}}+\left(\frac{2}{a} X_{0}-2\right) \frac{1}{\varepsilon_{1}}>0 . \text { In this case we obtain }
$$

$$
X_{0}>\frac{1}{2} a
$$

$$
\left(\frac{2}{a} X_{0}-2\right) \frac{1}{\varepsilon_{1}}<\xi \sin \xi-\frac{1}{\varepsilon_{1}} \cos \xi .
$$


Where $\xi$ - root of the equation $\xi=-\frac{1}{\varepsilon_{1}} \operatorname{tg} \xi$ for $\frac{1}{\varepsilon_{1}} \neq 0$, $0<\xi<\pi$. According to condition $\varepsilon_{1} \xi=-\operatorname{tg} \xi, y_{1}=\varepsilon_{1} \xi$, $y_{2}=-\operatorname{tg} \xi$.

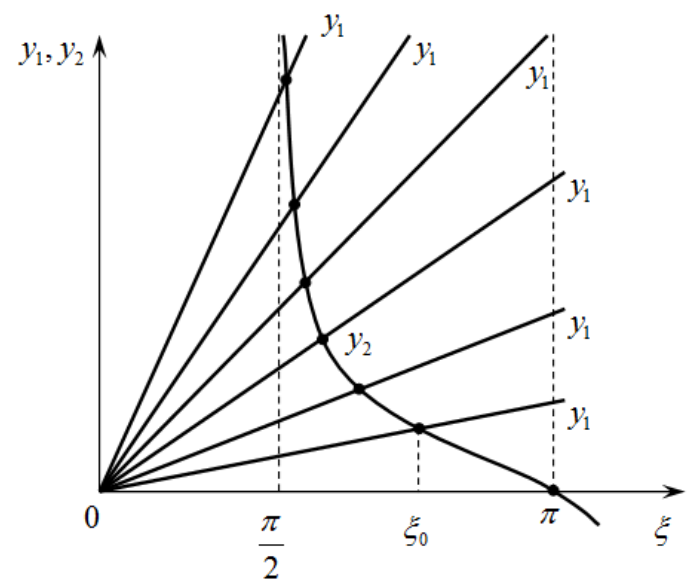

Figure 1. Fulfillment of the third condition of the Hayes criterion

$$
\begin{gathered}
\varepsilon_{1} \rightarrow \infty, \xi \rightarrow \frac{\pi}{2}, \\
\left(\frac{2}{a} X_{0}-2\right) \frac{1}{\varepsilon_{1}}<\frac{\pi}{2} .
\end{gathered}
$$

Due to $\varepsilon_{1} \rightarrow \infty$ is $\frac{1}{\varepsilon_{1}} \approx 0$. Herewith

$$
\frac{\pi}{2}>0
$$

1.3.2) $\varepsilon_{1} \rightarrow 0, \xi \rightarrow \pi$

$$
\left(\frac{2}{a} X_{0}-2\right) \frac{1}{\varepsilon_{1}}<\frac{1}{\varepsilon_{1}}
$$

By simplifying $\left(\frac{2}{a} X_{0}-2\right) \frac{1}{\varepsilon_{1}}<\frac{1}{\varepsilon_{1}}$, we get

$$
X_{0}<1 \frac{1}{2} a
$$

Reducing the conditions of Hayes criteria

$$
\begin{aligned}
& \frac{1}{\varepsilon_{1}}>-1 ; \\
& X_{0}>\frac{1}{2} a \\
& \frac{\pi}{2}>0 ; \\
& X_{0}<1 \frac{1}{2} a .
\end{aligned}
$$

To analyze the condition of the Hayes criterion, we have the following general conditions:

$$
\frac{1}{2} a<X_{0}<1 \frac{1}{2} a \text {. }
$$

We continue the analysis of (6) using the conditions of Hayes' criterion, as in (5), and it is necessary to arrive at the transcendental equation $(\lambda+a) e^{\lambda}+b=0$.

$$
\left(\lambda+\frac{1}{\varepsilon_{2}}\right) e^{\lambda}+\left(\frac{2}{b} \frac{Y_{0}^{2}}{X_{0}}-1\right) \frac{1}{\varepsilon_{2}}=0 .
$$

For the (9) we apply the condition of the Hayes criterion:

$$
\frac{1}{\varepsilon_{2}}>-1
$$

Given the positive value of the general parameters (1), then $\frac{1}{\varepsilon_{2}}>-1$ is satisfied.

$$
\begin{aligned}
& \frac{1}{\varepsilon_{2}}+\left(\frac{2}{b} \frac{Y_{0}^{2}}{X_{0}}-1\right) \frac{1}{\varepsilon_{2}}>0 \text {. Wherein } \\
& \frac{Y_{0}^{2}}{X_{0}}>0 . \\
&\left(\frac{2}{b} \frac{Y_{0}^{2}}{X_{0}}-1\right) \frac{1}{\varepsilon_{2}}<\xi \sin \xi-\frac{1}{\varepsilon_{2}} \cos \xi .
\end{aligned}
$$

Where $\xi$ - root of the equation $\xi=-\frac{1}{\varepsilon_{2}} \operatorname{tg} \xi$ at $0<\xi<\pi$, if $\frac{1}{\varepsilon_{2}} \neq 0$. According to condition $\varepsilon_{2} \xi=-\operatorname{tg} \xi, y_{1}=\varepsilon_{2} \xi$, $y_{2}=-\operatorname{tg} \xi$.

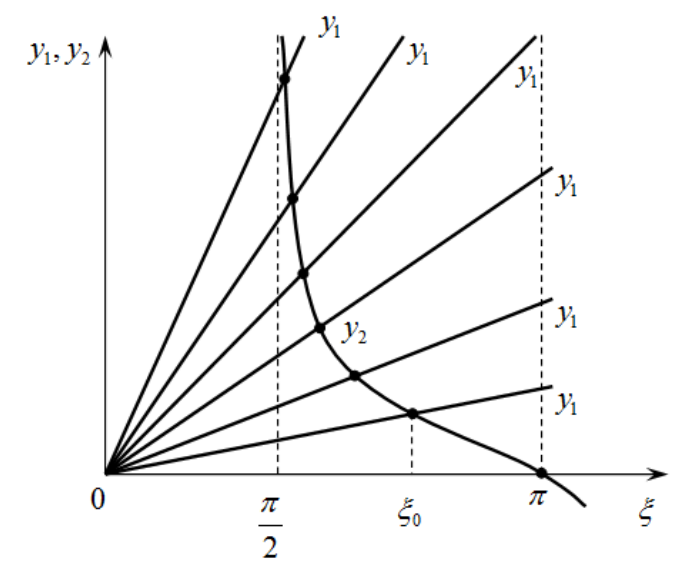

Figure 2. Fulfillment of the third condition of the Hayes criterion.

$$
\varepsilon_{2} \rightarrow 0, \xi \rightarrow \frac{\pi}{2}
$$




$$
\left(\frac{2}{b} \frac{Y_{0}^{2}}{X_{0}}-1\right) \frac{1}{\varepsilon_{2}}<\frac{\pi}{2}
$$

Due to $\varepsilon_{2} \rightarrow \infty$ is $\frac{1}{\varepsilon_{2}} \approx 0$. Herewith

$$
\begin{gathered}
\frac{\pi}{2}>0 . \\
\varepsilon_{2} \rightarrow \infty, \xi \rightarrow \pi, \\
\left(\frac{2}{b} \frac{Y_{0}^{2}}{X_{0}}-1\right) \frac{1}{\varepsilon_{2}}<\frac{1}{\varepsilon_{2}} .
\end{gathered}
$$

By simplifying $\left(\frac{2}{b} \frac{Y_{0}^{2}}{X_{0}}-1\right) \frac{1}{\varepsilon_{2}}<\frac{1}{\varepsilon_{2}}$, we get

$$
\frac{Y_{0}^{2}}{X_{0}}<b .
$$

Thus, we give the condition of the Hayes criterion:

$$
\begin{aligned}
& \text { 2.1) } \frac{1}{\varepsilon_{2}}>-1 \\
& \text { 2.2) } \frac{Y_{0}^{2}}{X_{0}}>0 \\
& \text { 2.3.1) } \frac{\pi}{2}>0 \\
& \text { 2.3.2) } \frac{Y_{0}^{2}}{X_{0}}<b .
\end{aligned}
$$

To analyze the condition of the Hayes criterion, we have the following general conditions:

$$
0<\frac{Y_{0}^{2}}{X_{0}}<b .
$$

Thus, if the fulfillment of the general conditions of the Hayes criterion (8) and (10) is then stable to the equilibrium position (1). Otherwise, it is not stable. This describes that you can observe the following regimes of functioning of the regulatory of the interrelated activity of the molecular genetic systems of hepatocyte and HBV: Poincare type limit cycles, chaos and "black hole" effects.

To carry out computational experiments, we created a computer model on the basis of equations (1) using the Runge-Kutta method $[15,16]$. The following expressions are used to perform the calculation [17]:

$$
\begin{aligned}
& X\left(t_{i}-1\right)=\left\{\begin{array}{l}
X\left(t_{0}\right) \text { at } t_{i}-1<t_{0} ; \\
\left(\sigma_{i}^{\prime \prime} X\left(t_{\mathbf{j}}\right)+\sigma_{i}^{\prime} X\left(t_{\mathrm{j}+1}\right)\right) / h, \\
\sigma_{i}^{\prime \prime}=\left(t_{i}-t_{j}\right)-1, \sigma_{i}^{\prime}=h-\sigma_{i}^{\prime \prime} \\
\text { at } t_{j}<t_{i}-1<t_{j+1} ;
\end{array}\right. \\
& j=0,1, \ldots, i ; i=0,1, \ldots, N,
\end{aligned}
$$

and

$$
\begin{aligned}
& Y\left(t_{i}-1\right)=\left\{\begin{array}{l}
Y\left(t_{0}\right) \text { at } t_{i}-1<t_{0} ; \\
\left(\sigma_{i}^{\prime \prime} Y\left(t_{\mathbf{j}}\right)+\sigma_{i}^{\prime} Y\left(t_{\mathbf{j}+1}\right)\right) / h, \\
\sigma_{i}^{\prime \prime}=\left(t_{i}-t_{j}\right)-1, \sigma_{i}^{\prime}=h-\sigma_{i}^{\prime \prime} \\
\text { at } t_{j}<t_{i}-1<t_{j+1} ;
\end{array}\right. \\
& j=0,1, \ldots, i ; i=0,1, \ldots, N .
\end{aligned}
$$

The values of $X(t-1)$ and $Y(t-1)$ in the mathematical model of the regulatory of the hepatocyte and HBV at the molecular-genetic level can be calculated using expressions (11) and (12).

\section{Results}

Computational experiments on the quantitative analysis of the regulatory of the hepatocyte and HBV show the presence following regime:

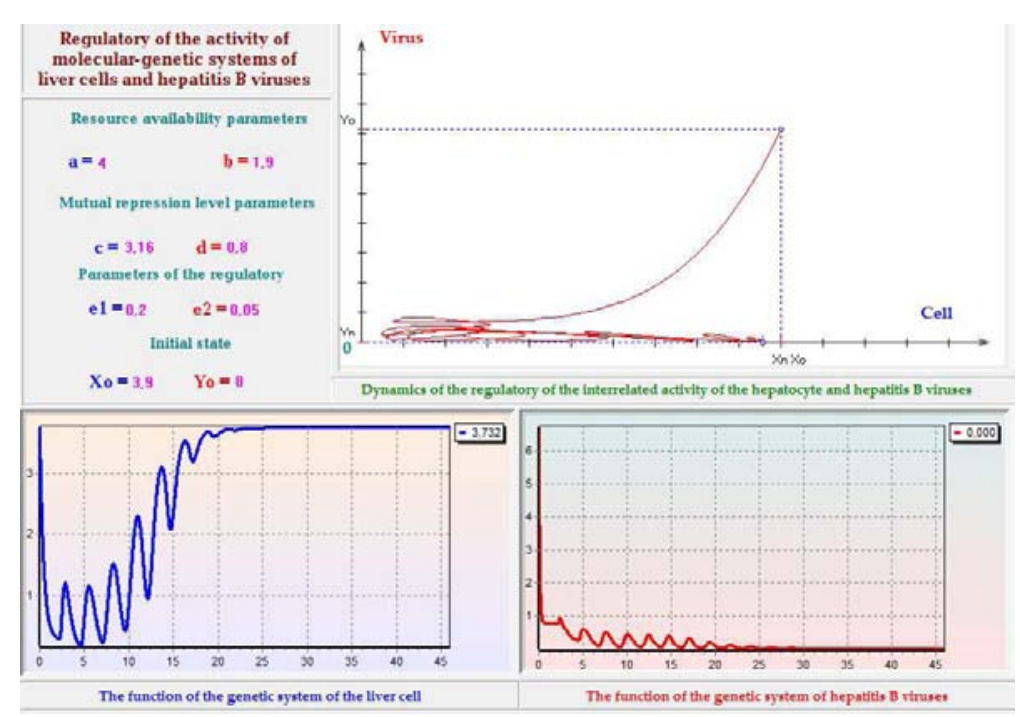

Figure 3. The regime of dominant functioning of the regulatory of hepatocyte and hepatitis B viruses. 
Figure 3 shows the dominant functioning of the genetic system of the hepatocyte with the following values of the parameters $\left(\varepsilon_{1}=0.2, \varepsilon_{2}=0.05, a=4, b=1.9, c=3.16, d=0.8\right.$ and $X_{0}=3.9, Y_{0}=8$ ), thus the activation of only the molecular genetic system of the hepatocyte with loss of HBV activity is described. The new virus will stop growing in the liver cell and the liver will be healthy.

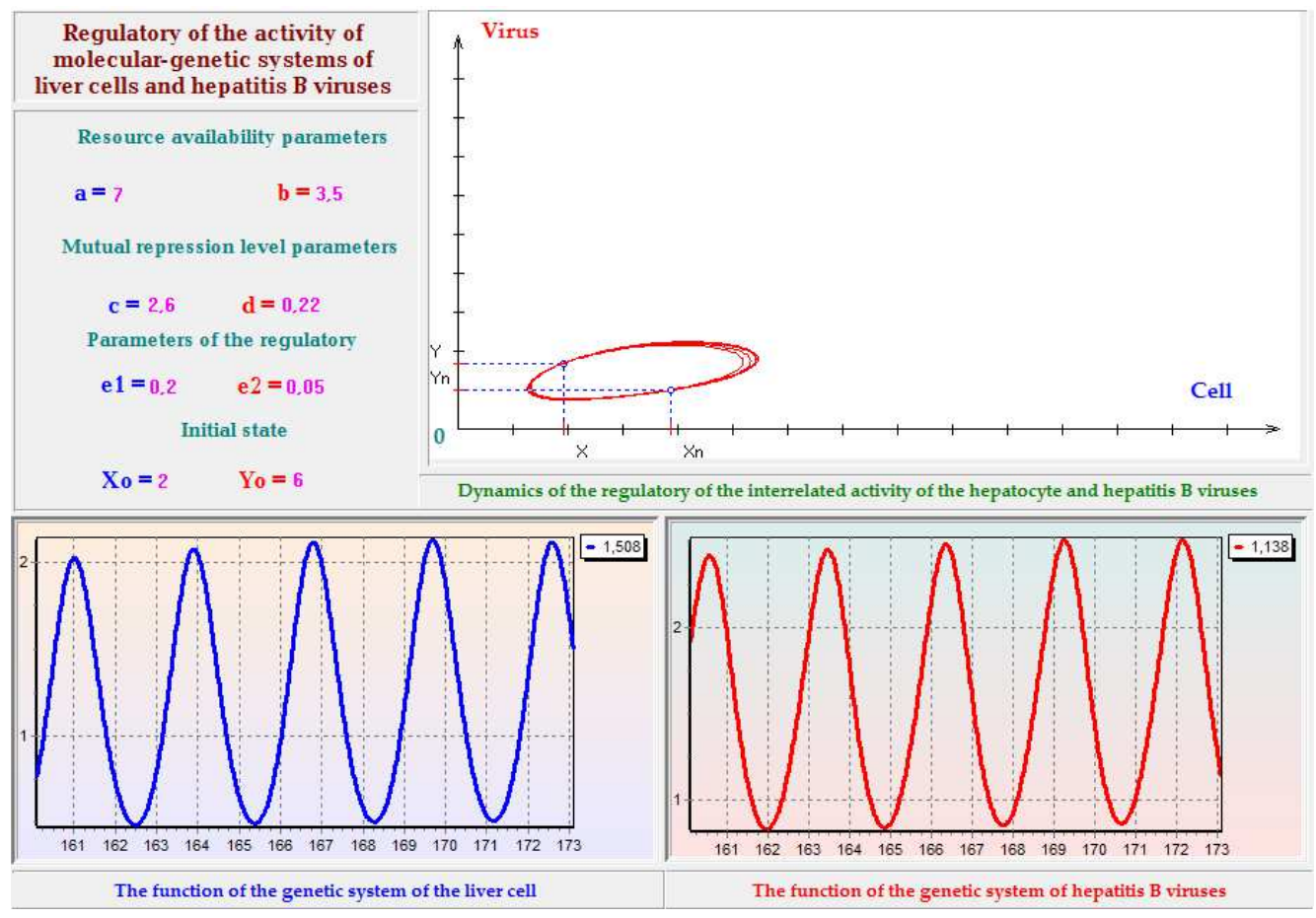

Figure 4. The regime of the limit cycle of regulatory of hepatocyte and hepatitis B viruses.

Figure 4 shows the limit cycle of the functioning of the genetic system of the hepatocyte with the following values of the parameters $\left(\varepsilon_{1}=0.2, \varepsilon_{2}=0.05, a=7, b=3.5, c=2.6, d=0.22\right.$ and $\left.X_{0}=2, Y_{0}=6\right)$. This describes the activation of the molecular genetic system of hepatocyte and HBV, as well as the onset of an infectious disease in the liver.

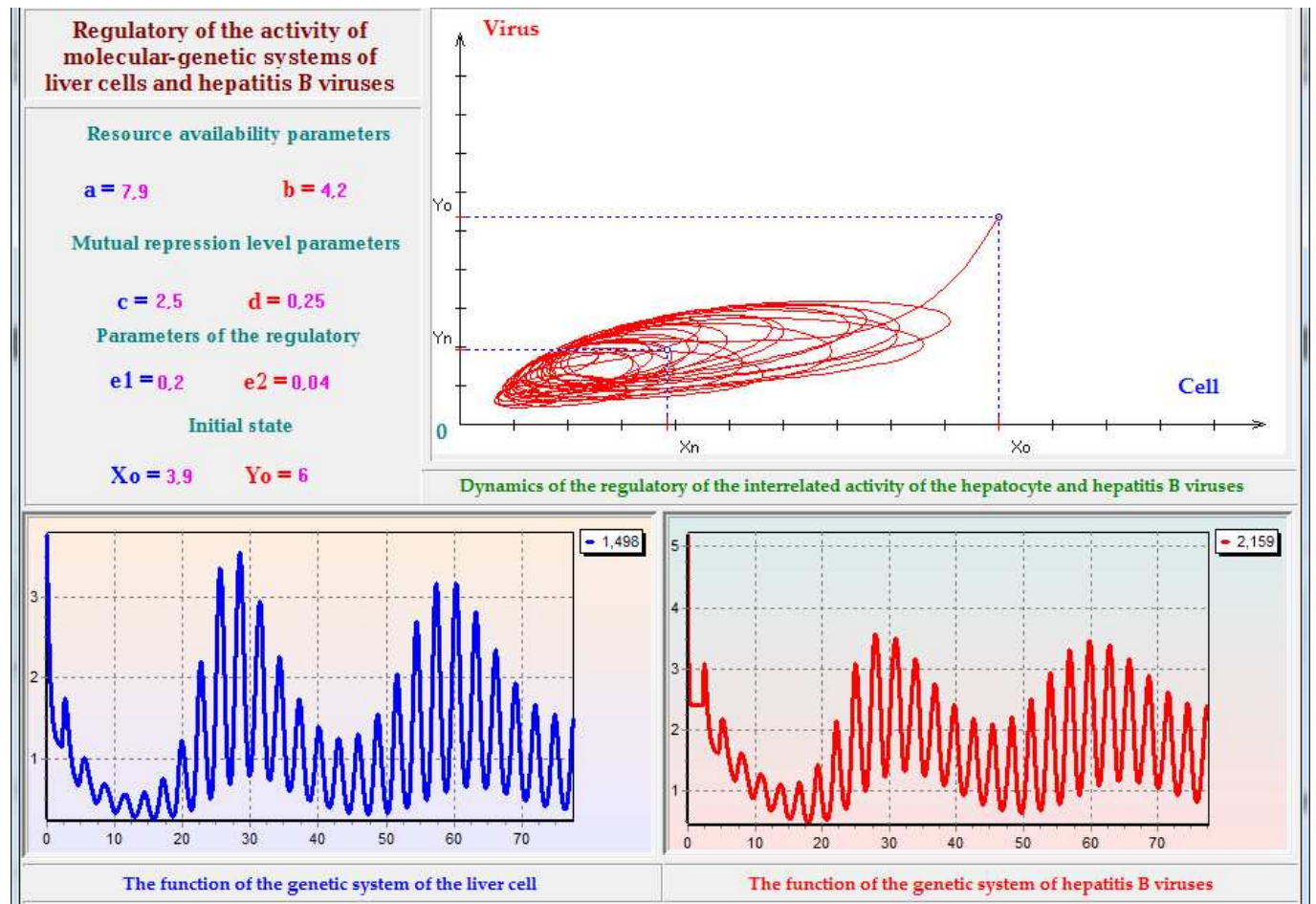

Figure 5. The regime of the chaos regulatory of hepatocyte and hepatitis $B$ viruses. 
Figure 5 shows the regime of the chaos regulatory of molecular genetic systems of liver cell and hepatitis B viruses with the following values of parameters $\left(\varepsilon_{1}=0.2, \varepsilon_{2}=0.04, a=7.9, b=4.2, c=2.5, d=0.25\right.$ and $\left.X_{0}=3.9, Y_{0}=6\right)$. The results in the figure show that the irregular functioning of the molecular genetic systems of the liver cell and HBV, and this describes the active infectious disease of viral hepatitis B in the liver.

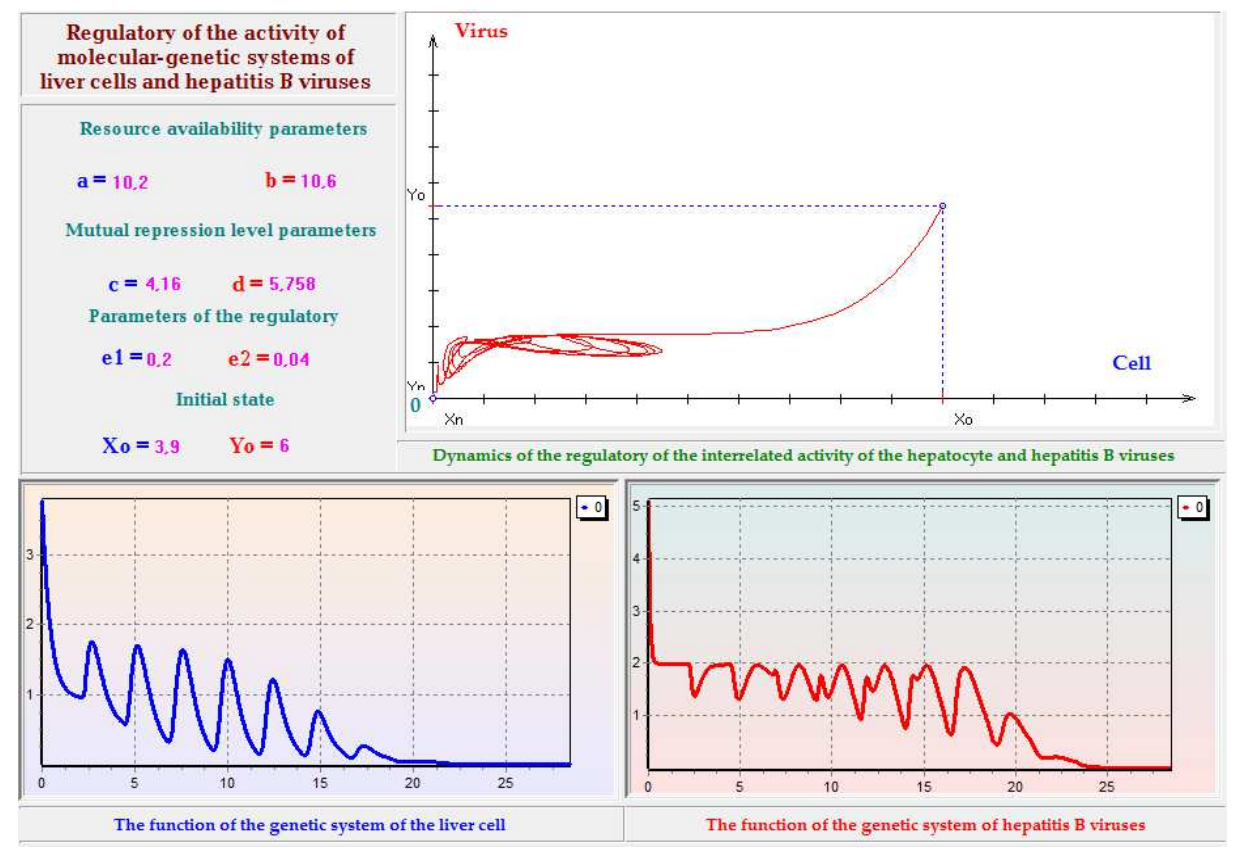

Figure 6. The regime of the Black hole"regulatory of hepatocyte and hepatitis B viruses.

In figure 6 shows the regime of the "black hole" regulatory of molecular genetic systems of liver cell and hepatitis B viruses with the following values of parameters $\left(\varepsilon_{1}=0.2\right.$, $\varepsilon_{2}=0.04, \quad a=10.2, \quad b=10.6, \quad c=4.16, \quad d=5.758$ and $X_{0}=3.9, Y_{0}=6$ ). The obtained results in the figure show that the functioning of the molecular genetic systems of the liver cell and HBV tends to zero and destroys the body.

\section{Conclusions}

Thus, the developed mathematical and computer models for the study of the functioning of the hepatocyte and HBV regulatory mechanisms allow one to assess the state of the interrelated activity of the molecular genetic systems of the liver cell and hepatitis B viruses; Establish the molecular genetic basis of pathogenesis; To assess and predict the occurrence of characteristic stages of the course of the disease with viral hepatitis B.

Computational experiments can be performed using the computer model developed. Based on the results of computational experiments, it is possible to develop a parametric portrait of the functioning of regulatory mechanisms of the interrelated activity of hepatocyte and hepatitis $\mathrm{B}$ viruses.

The parametric portrait includes all the regimes of the regulatory of liver cell and hepatitis $B$ viruses. The parametric portrait allows analyzing the regimes of the interrelated activity of liver cell and hepatitis B viruses at the molecular genetic level.

\section{References}

[1] World Health Organization (2016). Fact Sheet, July, Available at http://www.who.int/topics/hepatitis/en/.

[2] Perova I. G., Khludeeva O. I. (2015). Management of the development of infectious diseases. System of information boxes. 1 (126): 174-176.

[3] Lancet 2017.390: 1151-210. http://www.thelancet.com/journals/lancet/article/PIIS01406736 (17)32152-9/fulltext.

[4] Sunbul M. (2014). Hepatitis B virus genotypes: global distribution and clinical importance. World J. Gastroenterology. 20 (18): 5427-5434.

[5] Abu O., Onalo S. E. (2017). Numerical Analysis of a Mathematical Model of Hepatitis B Virus Transmission Dynamics in the Presence of Vaccination and Treatment. Journal of Scientific and Engineering Research. 4 (9): 295-310.

[6] Moneim I. A., Khalil H. A. (2015). Modeling and Simulation of the Spread of HBV Disease with Infectious Latent. Applied Mathematics. 6: 745-753. http://dx.doi.org/10.4236/am.2015.65070

[7] Hidirov B. N., Turgunov A. M. (2012). Modeling of molecular genetics mechanisms of control of viral hepatitis B. Uzbek journal of the Problems of Informatics and Energetics. 2-3: 13-18.

[8] Saidalieva M., Hidirova M. B., Turgunov A. M. (2014). Areas of homogeneous solutions of the equations of the mathematical model of the regulatory of liver in hepatitis B. Uzbek journal of the Problems of Informatics and Energetics. 6: 3-8. 
[9] Hidirova M. B., Turgunov A. M. (2015). Computer modeling of infectious disease with viral hepatitis B using information technologies. Materials of the XV International Scientific and Methodical Conference "Informatics: Problems, Methodology, Technologies". 1: 478-481.

[10] Hidirova M. B., Saydalieva M., Turgunov A. M. (2016). Analysis of the molecular and genetic mechanisms of liver cells under a load of its viruses hepatitis "B". Scientific articles International scientific-practical conference "INNOVATION-2016": 268-269.

[11] Turgunov A. M. (2017). On the modeling of regulatory of the liver cell and hepatitis B viruses. Scientific journal "Problems of computational and applied mathematics". 4 (10): 53-62.

[12] Turgunov A. M. (2017). Characteristic regimes of the behavior of solutions of the regulator equations of the "Hepatocyte-HBV" system. Materials of the XVII International Scientific and Methodical Conference "Informatics: Problems, Methodology, Technologies". 2: 446450 .
[13] Saidalieva M., Hidirova M. B., Turgunov A. M. (2015). Modeling of the regulatory of the liver cell in the quasistationary state of the hepatitis B virus. Scientific - technical and information-analytical journal TUIT - TUIT BULLETIN. 3 (35)/2015: 160-165.

[14] Hale J. (1984). Theory of Functional Differential Equations. M. The World. 421.

[15] Pimenov V. G. (2008). Functional-differential equations in biology and medicine. Tutorial. Ekaterinburg. 92.

[16] Hall G., Watt J. M. (1976). Modern Numerical Methods for Ordinary Differential Equations. Clarendon Press. Oxford. 312.

[17] Turgunov A. (2017). Analysis of the regulatory of the liver cell and hepatitis B viruses using a computer model. Collection of reports of the republican scientific and technical conference "The importance of information and communication technologies in the innovative development of real sectors of the economy". 1: 263-265. 